\title{
Tacrolimus as a Possible Trigger of Tachycardias in a Heart-Transplanted Patient with Severe Diarrhea
}

\author{
Christian Blockhaus ${ }^{1,2, *}$, Peter Waibler ${ }^{1}$, Jan Gülker ${ }^{1,2}$, Bülent Köktürk²,2, Alexander Bufe ${ }^{1,2}$, Dong-In Shin ${ }^{1}$, and Heinrich Klues ${ }^{1}$ \\ ${ }^{1}$ Heart Centre Niederrhein, Department of Cardiology, Helios Clinics Krefeld, Germany \\ ${ }^{2}$ Witten-Herdecke University, Germany
}

*Corresponding author: Christian Blockhaus, Heart Centre Niederrhein, Department of Cardiology, Helios Clinics Krefeld, Lutherplatz 40, 47805 Krefeld, Germany, Tel: +492151322702; E-mail: christian.blockhaus@helios-gesundheit.de

Received: 13 Nov, 2020 | Accepted: 27 Nov, 2020 | Published: 04 Dec, 2020

Citation: Blockhaus C, Waibler P, Gülker J, Köktürk B, Bufe A, et al. (2020) Tacrolimus as a Possible Trigger of Tachycardias in a Heart-Transplanted Patient with Severe Diarrhea. J Heart Health 7(1): dx.doi.org/10.16966/2379-769X.158

Copyright: (C) 2020 Blockhaus C, et al. This is an open-access article distributed under the terms of the Creative Commons Attribution License, which permits unrestricted use, distribution, and reproduction in any medium, provided the original author and source are credited.

\section{Abstract}

Tacrolimus is a broadly used immunosuppressive agent in organ transplanted patients. It is known that it may induce arrhythmias. We here report on a heart transplanted patient with supraventricular and ventricular arrhythmias with an elevated tacrolimus trough serum level due to severe diarrhea.

Keywords: Tacrolimus; Arrhythmia; Heart transplantation; Graft rejection

\section{Introduction}

In organ-transplanted patients, tacrolimus is a broadly used immunosuppressive agent with a small therapeutic range; the serum level is measured as trough level. One of the side effects may be diarrhea but also arrhythmias. We here report on a case of tacrolimus induced atrial and ventricular tachycardia in a heart-transplanted patient with severe diarrhea.

\section{Case Report}

A 53-year-old male presented to our hospital in 2020 due to dyspnea and sudden onset of a symptomatic tachycardia two days earlier. He had suffered from myocarditis decades ago with severely reduced heart function. In 2013, he underwent a biatrial orthotopic heart transplantation. The patient was under immunosuppressive medication with mycophenolate mofetil (360 mg twice daily) and tacrolimus ( $3 \mathrm{mg}$ twice daily) and presented periodically to our outpatient clinic. The target trough level of tacrolimus for the patient was $5-8 \mathrm{ng} / \mathrm{ml}$ according to the guidelines [1]. Steroid therapy had been stopped at least 5 years ago. To date, there had been no documentation of any arrhythmia e.g. atrial fibrillation or congestive heart failure. The electrocardiogram at admission showed an atrial tachycardia with 185 beats per minute (Figure 1).

The tacrolimus serum trough level was increased with $23.1 \mathrm{ng} / \mathrm{ml}$, furthermore the patient showed a highly elevated Brain Natriuretic Peptide (BNP) of $23761 \mathrm{pg} / \mathrm{ml}$ and an acute on chronic renal failure (actual creatinine $2.31 \mathrm{mg} / \mathrm{dl}$ at admission; previously: $1.3-1.4 \mathrm{mg} / \mathrm{dl}$ ). The patient suffered since many years from a diabetic nephropathy.
Sodium was $138 \mathrm{mml} / \mathrm{l}$, calcium $2.13 \mathrm{mml} / \mathrm{l}$ and potassium in a normal range with $4.7 \mathrm{mmol} / \mathrm{l}$. Hemoglobin was slightly decreased with $10.4 \mathrm{~g} / \mathrm{dl}$ which was stable compared to previous blood samples. During telemetry observation, the patient also showed episodes of paroxysmal atrial fibrillation (not shown), sinus rhythm (Figure 2 ) and non-sustained ventricular tachycardias (Figure 3). Initially, we withheld the administration of tacrolimus. We furthermore performed a myocardial biopsy where acute transplant rejection was excluded, and a coronary angiography which excluded a transplant vasculopathy. After exclusion of intracardiac thrombus using transesophageal echocardiography, we started administration of amiodarone. As the patient reported an episode of severe diarrhea of about four days before presentation to our hospital, we carefully administered intravenous fluid. The patient reported no selftreatment or consultation of other medical services before admission. The echocardiography revealed normal left ventricular and right ventricular function without signs of valvular dysfunction. Within three days, there were no more documented arrhythmias, the creatinine decreased to previous levels and BNP decreased to 1254 $\mathrm{pg} / \mathrm{ml}$. The patient was discharged with a normal tacrolimus level (7.4 $\mathrm{ng} / \mathrm{ml}$ ) in stable conditions and without amiodarone. Till today, two months later, no further documented arrhythmia occurred.

\section{Discussion}

Emerging tachycardias in patients after heart transplantation may have different reasons. Mostly, patients present with macroreentrant tachycardias which may be isthmus dependent flutter or scar related tachycardia [2]. Atrial fibrillation mostly occurs in the 


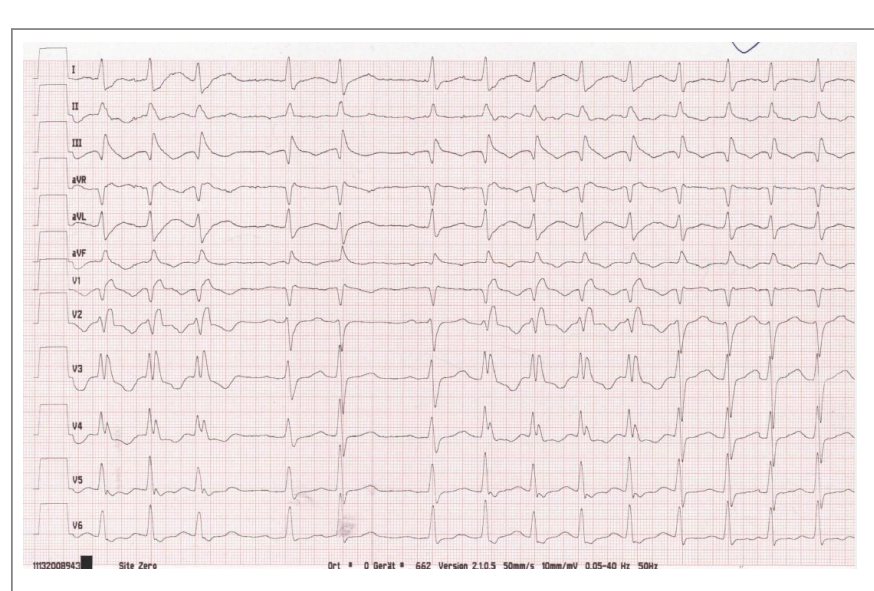

Figure 1: Electrocardiogram of the atrial tachycardia $(50 \mathrm{~mm} / \mathrm{s})$.

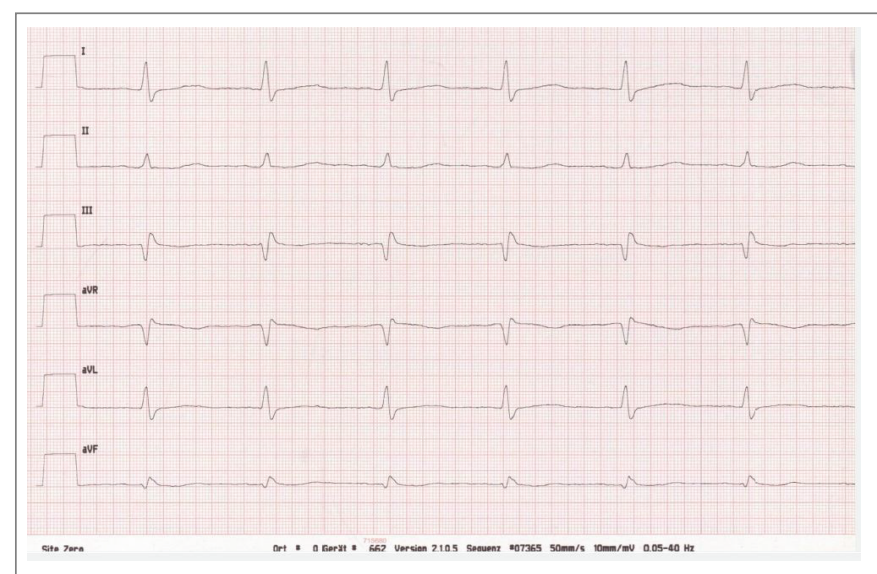

Figure 2: Electrogram of sinus rhythm $(50 \mathrm{~mm} / \mathrm{s})$.

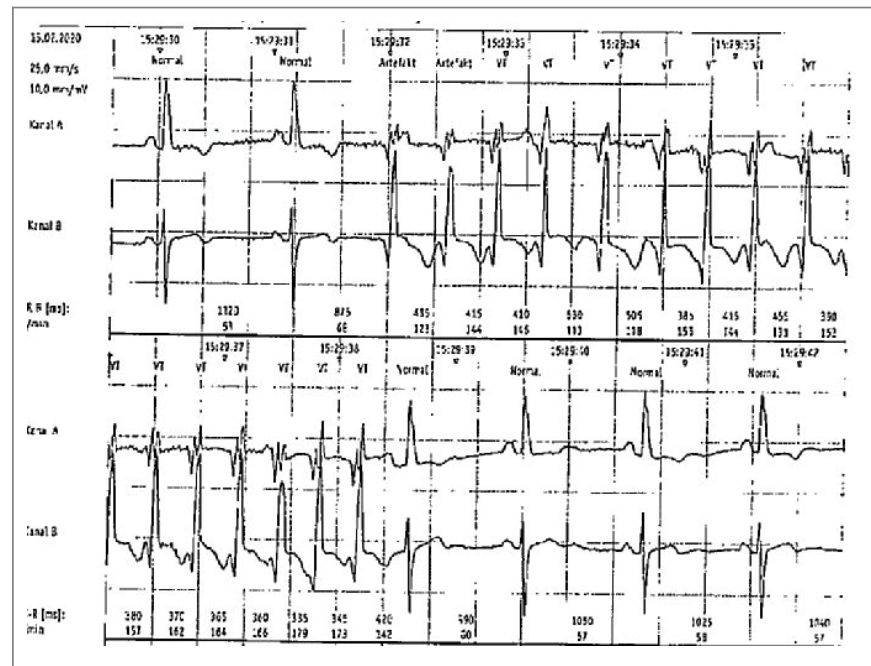

Figure 3: Telemetry observation of nsVT.

early postoperative phase. The overall incidence after transplantation is approximately $10 \%$ as presented by Chokesuwattanaskul $\mathrm{R}$, et al. in their meta-analysis and review [3]. Onset of post-transplant atrial fibrillation is associated with poorer outcomes [3,4]. There are two operation techniques, namely, the bicaval and biatrial heart transplantation. The biatrial technique leaves more of the recipients left atrium, causes more scar tissue, and is therefore more often associated with the onset of atrial fibrillation or scar related tachycardias [3,5]. As our reported patient suffered from acute onset of atrial fibrillation, atrial tachycardia and non-sustained VTs, however, we do not think that this is related to the operation technique.

Ventricular Tachycardias (VT) in the early postoperative period may be sign of acute graft rejection. Onset of VTs years after transplantation may be a sign of graft rejection or a severe transplant vasculopathy. In those cases, a coronary angiography and myocardial biopsy have to be performed which was done in our case [5].

Tacrolimus is a frequently used immunosuppressive agent in transplanted patients. It is a macrolide and a potent calcineurin inhibitor metabolized via cytochromeP3A4. Other agents metabolized by CYP3A4 may interact with tacrolimus leading to increased or decreased plasma levels. In patients treated with MMF and tacrolimus, diarrhea is a typical adverse effect. Furthermore, tacrolimus has a prokinetic effect. In our case, the patient had suffered from severe diarrhea for several days. In addition, he only drank little water due to nausea. It is known that severe diarrhea may lead to increased trough levels of tacrolimus [6]. The loss of fluid may also be the trigger of acute on chronic kidney failure which may itself lead to increasing tacrolimus levels. Severe diarrhea provokes an intestinal damage with increased absorption and decreased metabolism of tacrolimus [7]. The increased BNP levels may be explained by the acute on chronic kidney failure and in addition the tachycardia.

Even though the incidence is rare, tacrolimus may induce arrhythmias even if the mechanism remains unclear [8,9]. It may be necessary to pause the administration, until normal serum trough levels are achieved and tachycardias cease. In our case, we administered amiodarone for several days until sinus rhythm was observed continuously. Amiodarone and tacrolimus may both lead to QT-prolongation andthe QT-interval has to be monitored carefully during application. A limiting factor of this case report is the administration of amiodarone when the patient developed nonsustained VTs. The cessation of the tachycardias may be more likely due to amiodarone that was administered for six days until decreasing tacrolimus trough levels. However, as the patient is now free of any arrhythmia two months later with normal tacrolimus trough levels we suppose the increased trough levels as the most likely reason for theses arrhythmias.

\section{Conclusion}

Heart-transplanted patients with new onset of any tachycardia have to be monitored carefully. Graft rejection and transplant vasculopathy have to be beared in mind just as changes in serum electrolyes. Immunosuppressive agents have a small therapeutic range and may be influenced by many factors like other medicaments, acute kidney failure, diarrhea or inaccurate intake. We here report on elevated tacrolimus trough levels as a potential trigger of arrhythmias.

\section{Conflict of Interest}

The authors state that they have no conflict of interest.

\section{References}

1. Costanzo MR, Dipchand A, Starling R, Anderson A, Chan M, et al. (2010) The International Society of Heart and Lung Transplantation Guidelines for the care of heart transplant recipients. J Heart Lung Transplant 29: 914-956.

2. Vaseghi M, Boyle NG, Kedia R, Patel JK, Cesario DA, et al. (2008) Supraventricular Tachycardia after Orthotopic Cardiac Transplantation. J Am Coll Cardiol 51: 2241-2249. 
3. Chokesuwattanaskul R, Bathini T, Thongprayoon C, Preechawat S, O'Corragain OA, et al. (2018) Atrial Fibrillation Following Heart Transplantation: A Systematic Review and Meta-Analysis of Observational Studies. J Evid Based Med 11: 261-271.

4. Pavri BB, O'Nunain SS, Newell JB, Ruskin JN, William G (1995) Prevalence and Prognostic Significance of Atrial Arrhythmias after Orthotopic Cardiac Transplantation. J Am Coll Cardiol 25: 1673-1680.

5. Thajudeen A, Stecker EC, Shehata M, Patel J, Wang X, et al. (2012) Arrhythmias after Heart Transplantation: Mechanisms and Management. J Am Heart Assoc 1: e001461.

6. Lee CH, Tasker N, La Hei E, Dutt S (2014) Raised Tacrolimus Level and Acute Renal Injury Associated with Acute Gastroenteritis in a Child Receiving Local Rectal Tacrolimus. Clin J Gastroenterol 7: 238-242.
7. Lemahieu W, Maes B, VerbekeK, Rutgeerts P, Geboes K, et al. (2005) Cytochrome P450 3A4 and P-glycoprotein Activity and Assimilation of Tacrolimus in Transplant Patients with Persistent Diarrhea. Am J Transplant 5: 1383-1391.

8. Kim BR, Shin HS, Jung YS, Rim H (2013) A Case of Tacrolimus-Induced Supraventricular Arrhythmia after Kidney Transplantation. Sao Paulo Med J 131: 205-207.

9. Nishimura M, Kim K, Uchiyama A, Fujino Y, Nishimura S, et al. (2002) Tacrolimus-induced Life-Threatening Arrhythmia in a Pediatric LiverTransplant Patient. Intensive Care Med 28: 1683-1684. 